\title{
Integrin Calculus: The Predictive Power of Vedolizumab Concentrations in IBD Therapy
}

\author{
Konstantinos Papamichael ${ }^{1} \cdot$ Adam S. Cheifetz $^{1}$
}

Published online: 29 March 2019

(c) Springer Science+Business Media, LLC, part of Springer Nature 2019

\begin{abstract}
Although biological therapies, including the monoclonal antibody directed against the $\alpha_{4} \beta_{7}$ integrin, vedolizumab, have revolutionized the treatment of patients with inflammatory bowel disease (IBD), response and remission to this therapy are not universal. Up to $30 \%$ of patients with Crohn's disease (CD) and ulcerative colitis (UC) do not respond at all, and up to $70 \%$ of initial responders only partially respond, with many patients losing response over time. Many of these undesired therapeutic outcomes can be explained by undetectable or low drug concentrations due to antibody formation or an increased non-immune drug clearance rate. Numerous studies suggest that higher serum biological drug concentrations are associated with a greater rate of favorable therapeutic outcomes [1]. Nonetheless, most of these studies relate to therapies directed against tumor necrosis factor (TNF), whereas exposure-outcome relationship studies remain limited for vedolizumab. Consequently, the time to assess drug concentrations and the related therapeutic drug window for vedolizumab remains undefined.

In this issue of Digestive Diseases and Sciences, Yarur et al. [2] recently added to knowledge regarding therapeutic drug monitoring (TDM) of vedolizumab. They performed a nicely designed single-center prospective cohort study that reported that serum vedolizumab concentrations obtained shortly after therapy initiation correlated with long-term steroid-free endoscopic remission. This study of 55 patients with IBD (CD, $n=25$, and UC, $n=30)$ and with active endoscopic disease in whom vedolizumab therapy was initiated demonstrated that patients achieving steroid-free endoscopic remission by week 52 of therapy had statistically significant higher serum vedolizumab concentrations at weeks 2 and 6 .
\end{abstract}

Adam S. Cheifetz

acheifet@bidmc.harvard.edu

1 Center for Inflammatory Bowel Diseases, Division of Gastroenterology, Beth-Israel Deaconess Medical Center, Harvard Medical School, 330 Brookline Ave., Rabb 425, Boston, MA 02215, USA
Additionally, they identified that vedolizumab concentration thresholds $\geq 23.2 \mu \mathrm{g} / \mathrm{ml}$ at week 2 and $\geq 19.8 \mu \mathrm{g} / \mathrm{ml}$ at week 6 were independently associated with endoscopic (odds ratio [OR] 8.8; 95\% confidence interval [CI] 2.6-29.7; $p<0.001$ ) and clinical (OR 6.8 [95\% CI 1.2-4], $p=0.033$ ) remission at week 52. Furthermore, they demonstrated a positive correlation between serum vedolizumab concentrations and serum albumin and an inverse correlation with C-reactive protein (CRP), fecal calprotectin, and body mass. Finally, immunogenicity was very low as only $3 / 55(5.5 \%)$ patients had detectable antibodies to vedolizumab through the 52 weeks of follow-up using a drug-tolerant homogeneous mobility shift assay.

These results are in line with previous studies, showing that higher vedolizumab concentrations, during both induction and maintenance treatments, are typically associated with superior therapeutic outcomes in patients with IBD (Table 1) [2-8]. The identification of clinically relevant vedolizumab thresholds is important as this would be the first step for applying both reactive and proactive TDMbased therapeutic algorithms. These algorithms for reactive TDM have been proven useful and cost-effective for clarifying the etiology and managing the loss of response to anti-TNF therapy, whereas proactive TDM is associated with superior clinical outcomes when compared to standard of care (empiric dose escalation and/or reactive TDM) for both infliximab and adalimumab.

This prospective, though rather small, study highlights the significance of TDM for optimizing vedolizumab therapy for IBD therapy during the early induction phase in order to achieve superior long-term outcomes. Similar to anti-TNF therapies, this study showed that TDM is likely even more clinically important for patients with a greater inflammatory burden, such as patients with high CRP and low albumin. Nevertheless, as the authors also stated, interventional studies testing higher drug doses during induction in order to improve therapeutic outcomes are needed. Before proactive TDM of vedolizumab can be widely implemented 
Table 1 Association of serum vedolizumab concentration thresholds with therapeutic outcomes in inflammatory bowel disease

\begin{tabular}{lllllll}
\hline IBD type & Study type & Time point & Threshold $(\mu \mathrm{g} / \mathrm{ml})$ & Therapeutic outcome & TDM assay & References \\
\hline $\mathrm{CD}$ & Retrospective & Induction (w2) & $>35.2$ & Biological remission (w6) & ELISA & [3] \\
$\mathrm{UC}$ & Retrospective & Induction (w2) & $>28.9$ & Clinical response (w14) & ELISA & [3] \\
$\mathrm{UC}$ & Retrospective & Induction (w2) & $>23.7$ & Mucosal healing (w14) & ELISA & [3] \\
$\mathrm{CD} / \mathrm{UC}$ & Prospective & Induction (w2) & $<24.5$ & Drug optimization (within w24) & ELISA & [4] \\
$\mathrm{CD} / \mathrm{UC}$ & Prospective & Induction (w2) & $\geq 23.2$ & Endoscopic remission (w52) & HMSA & [2] \\
$\mathrm{UC}$ & Retrospective & Induction (w6) & $>20.8$ & Clinical response (w14) & ELISA & [3] \\
$\mathrm{UC}$ & Post hoc analysis of RCT & Induction (w6) & $>37.1$ & Clinical remission (w14) & ELISA & [5] \\
$\mathrm{CD} / \mathrm{UC}$ & Prospective & Induction (w6) & $<18.5$ & Need for extended therapy & ELISA & [4] \\
$\mathrm{CD} / \mathrm{UC}$ & Prospective & Induction (w6) & $>27.5$ & Sustained clinical response & ELISA & [4] \\
$\mathrm{CD} / \mathrm{UC}$ & Prospective & Induction (w6) & $>18$ & Mucosal healing (within w54) & ELISA & [6] \\
$\mathrm{CD} / \mathrm{UC}$ & Retrospective & Induction (w6) & $>28$ & Sustained response & ELISA & [7] \\
$\mathrm{CD} / \mathrm{UC}$ & Prospective & Induction (w6) & $\geq 19.8$ & Clinical remission (w52) & HMSA & [2] \\
$\mathrm{UC}$ & Retrospective & Post-induction (w14) & $>12.6$ & Clinical response (w14) & ELISA & [3] \\
$\mathrm{UC}$ & Retrospective & Post-induction (w14) & $>17$ & Mucosal healing (w14) & ELISA & [3] \\
$\mathrm{UC}$ & Post hoc analysis of RCT & Post-induction (w14) & $>18.4$ & Clinical remission (w14) & ELISA & [5] \\
$\mathrm{CD}$ & Retrospective & Maintenance (w22) & $>13.6$ & Mucosal healing (w22) & ELISA & [3] \\
$\mathrm{CD}$ & Retrospective & Maintenance (w22) & $>12$ & Biological remission (w22) & ELISA \\
$\mathrm{UC}$ & Retrospective & Maintenance & $>25$ & Histological healing & [3] \\
$\mathrm{UC}$ & Post hoc analysis of RCT & Maintenance & $>12.7$ & Clinical remission (w52) & ELISA & [8] \\
\hline
\end{tabular}

ELISA enzyme-linked immunosorbent assay, HMSA homogeneous mobility shift assay, $w$ week, $C D$ Crohn's disease, $U C$ ulcerative colitis, $R C T$ randomized controlled trial, $R e f$ reference

${ }^{\mathrm{a}}$ GEMINI 1

in "real-life" clinical practice, several goals need to be achieved, including determining the optimal concentration range and therapeutic window to target, obtaining more data regarding specific IBD phenotypes that respond most favorably, defining the types of TDM assays used, and conducting prospective studies on the implementation of proactive TDM.

\section{Compliance with ethical standards}

Conflict of interest KP has nothing to disclose; ASC received a consultancy fee from Janssen, Abbvie, Takeda, Pfizer, Samsung, Arena, Bacainn, EMD Serono, Arsanis, Grifols, and Prometheus and research support from Inform Diagnostics.

\section{References}

1. Papamichael K, Cheifetz AS. Use of anti-TNF drug levels to optimise patient management. Frontline Gastroenterol. 2016;7:289-300.

2. Yarur AJ, Bruss A, Naik S, et al. Vedolizumab concentrations are associated with long-term endoscopic remission in patients. Dig Di Sci. (Epub ahead of print). https://doi.org/10.1007/s1062 0-019-05570-1.
3. Dreesen E, Verstockt B, Bian S, et al. Evidence to support monitoring of vedolizumab trough concentrations in patients with inflammatory bowel diseases. Clin Gastroenterol Hepatol. 2018;16:1937-1946.

4. Williet N, Boschetti G, Fovet M, et al. Association between low trough levels of vedolizumab during induction therapy for inflammatory bowel diseases and need for additional doses within 6 months. Clin Gastroenterol Hepatol. 2017;15:1750-1757.

5. Osterman MT, Rosario M, Lasch K, et al. Vedolizumab exposure levels and clinical outcomes in ulcerative colitis: determining the potential for dose optimization. Aliment Pharmacol Ther. 2019;49:408-418.

6. Yacoub W, Williet N, Pouillon L, et al. Early vedolizumab trough levels predict mucosal healing in inflammatory bowel disease: a multicentre prospective observational study. Aliment Pharmacol Ther. 2018;47:906-912.

7. Liefferinckx C, Minsart C, Cremer A, et al. Early vedolizumab trough levels at induction in inflammatory bowel disease patients with treatment failure during maintenance. Eur J Gastroenterol Hepatol. (Epub ahead of print). https://doi.org/10.1097/ meg.0000000000001356.

8. Pouillon L, Rousseau H, Busby-Venner et al. Vedolizumab trough levels and histological healing during maintenance therapy in ulcerative colitis. J Crohns Colitis. (Epub ahead of print). https:// doi.org/10.1093/ecco-jcc/jjz029.

Publisher's Note Springer Nature remains neutral with regard to jurisdictional claims in published maps and institutional affiliations. 